\title{
Multi-Hop Coherent Free-Space Optical Communications over Atmospheric Turbulence Channels
}

\author{
Sahar Molla Aghajanzadeh, Student Member, IEEE, and Murat Uysal, Senior Member, IEEE
}

\begin{abstract}
In this paper, we investigate multi-hop relaying as an efficient fading mitigation tool for coherent free-space optical (FSO) systems over atmospheric turbulence channels. We consider an FSO relaying system with decode-and-forward relay nodes and multiple heterodyne receivers with modal compensation. Based on a recently introduced statistical characterization for the combined effects of log-normal turbulence fading and modal compensation, we derive the outage probability and quantify the potential performance improvements through the derivation of diversity-multiplexing tradeoff (DMT) and diversity gain. Our outage analysis yields impressive power savings for multi-hop relaying even with a single-relay. In addition, the DMT analysis in practical signal-to-noise ratio (SNR) ranges demonstrates that multi-hop transmission scheme improves finite-SNR diversity gain throughout the range of the multiplexing gain.
\end{abstract}

Index Terms-Free-space optical systems, multi-hop transmission, finite-SNR diversity-multiplexing tradeoff, atmospheric turbulence-induced fading.

\section{INTRODUCTION}

$\mathbf{F}$ REE-SPACE optical (FSO) communication is a costeffective and high-bandwidth transmission technique deployed in unregulated spectrum [1]-[3]. A major performance limiting factor for FSO links is the atmospheric turbulenceinduced fading. It has been shown in [4]-[9] that multi-hop relaying brings impressive performance improvements in FSO systems with intensity modulation/direct detection (IM/DD). In comparison to IM/DD systems, coherent FSO systems are relatively more difficult to implement; however, they offer distinct advantages. By virtue of mixing the received signal with a strong local oscillator (LO) field, these systems have much better spatial and frequency selectivity compared to their non-coherent counterpart. The spatial selectivity property is particularly useful for applications where background noise, multiple access (or intentional) interference, and atmospheric turbulence are performance limiting factors [3]. Such advantages have recently motivated many researchers to work on

Paper approved by W. Shieh, the Editor for Optical Transmission and Switching of the IEEE Communications Society. Manuscript received February 3, 2010; revised October 8, 2010 and January 4, 2011.

S. M. Aghajanzadeh is with the Department of Electrical and Computer Engineering, University of Waterloo, Waterloo, Ontario, N2L 3G1, Canada (e-mail: smollaag@uwaterloo.ca).

M. Uysal is with the Faculty of Engineering, Özyeğin University, 34662, Istanbul, Turkey (e-mail: murat.uysal@ozyegin.edu.tr).

The work of M. Uysal is supported, in part, by the Marie Curie International Reintegration Grant (PIRG07-GA-2010-268318).

Digital Object Identifier 10.1109/TCOMM.2011.050911.100069 the theoretical and experimental aspects of coherent terrestrial FSO systems, see e.g., [10]-[12] and the references therein.

Aiming to take advantage of the inherent features of coherent detection, this paper investigates coherent FSO relaying for the first time in the literature to the best of our knowledge. Specifically, we consider a coherent multi-hop FSO relaying system employing decode-and-forward (DF) relay nodes and multiple heterodyne receivers with modal phase compensation [13]. Using a recently introduced statistical characterization [14] for the combined effects of log-normal turbulence fading and modal compensation, we take into account the impact of both atmospheric turbulence-induced amplitude and phase fluctuations on the coherent receiver. We derive outage probability and diversity-multiplexing tradeoff (DMT) [15] expressions for coherent relay-assisted FSO system under consideration. Our outage probability analysis demonstrates that an impressive performance improvement of $31.5 \mathrm{~dB}$ is possible with the use of a single relay at a target outage probability of $10^{-8}$. Our DMT analysis further demonstrates possible diversity gains throughout the range of multiplexing gain.

The rest of the paper is organized as follows: In Section II, we introduce the system model. In Section III, we derive the outage probability for the system under consideration. In Section IV, based on the outage probability, we obtain the finite signal-to-noise ratio (SNR) DMT expression and the finite SNR diversity gain for a fixed transmission rate. In Section V, we present numerical results and finally conclude in Section VI.

\section{SySTEM Model}

We consider a coherent multi-hop FSO relaying system in which the source signal arrives at the destination via a sequence of $M+1$ hops through $M$ intermediate relays. The nodes in the network are serially indexed from 0 to $M+1$ where $k=0$ and $k=M+1$ refer to the source and the destination, respectively. The link range $Z_{S D}$ (i.e., distance from the source to the destination) is given by $Z_{S D}=\sum_{k=1}^{M+1} Z_{k}$ where $Z_{k}, k=1,2, \ldots, M+1$, is the length of the $k^{\text {th }}$ hop. All nodes are equipped with single transmitters and each has transmitoptical power of $P_{s}=P_{t} /(M+1)$ where $P_{t}$ is the total power budget of the system. Relay and destination nodes have more than one heterodyne receiver (not necessarily the same number) to further exploit receive diversity. Let the $k^{\text {th }}$ node, $1 \leq k \leq M+1$, be equipped with $m_{k}$ parallel heterodyne 
receivers. The aperture diameter of each receiver located in the $k^{\text {th }}$ node is given by $D_{k}=D / \sqrt{m_{k}}$ where $D$ is the total receive-aperture diameter. Receive apertures are separated by more than the channel coherence length to ensure independent fading channels.

Each relay first combines the diversity branches using maximum ratio combiner. Then, it decodes the transmitted information and retransmits it to the next relay (or to the destination in the last hop). The noise term at each receiver output is dominated by LO shot noise and can be modeled as additive white Gaussian noise (AWGN) [1]. Consequently, the intermediate frequency component of the output signal at the $k^{\text {th }}$ node after heterodyning and diversity combining is given by $y_{k}(t)=x_{k}(t)+n_{k}(t)$ where $n_{k}(t)$ is the noise term and the information carrying part $x_{k}(t)$ is given by [16]

$$
\begin{aligned}
x_{k}(t)=2 \Re A_{L} & \frac{S_{r}}{m_{k}} \sqrt{\sum_{i=1}^{m_{k}}\left|\alpha_{k, i}\right|^{2}} u_{k}(t) \\
& \times \cos \left[2 \pi\left(f_{c}-f_{L}\right) t+\theta_{k}(t)-\theta_{L}\right] .
\end{aligned}
$$

Here, $\Re$ is the photodetector responsivity [17], $f_{c}$ is the optical carrier frequency, and $S_{r}=\pi D^{2} / 4$ is the receive-aperture area. $A_{L}, f_{L}$, and $\theta_{L}$ represent the amplitude, frequency, and phase of the LO field, respectively. $u_{k}(t) e^{j \theta_{k}(t)}$ is the complex envelope of the received modulation signal.

In (1), $\alpha_{k, i}$ denotes the effective fading coefficient (after heterodyning) of the $i^{\text {th }}$ diversity branch in the $k^{\text {th }}$ hop. Considering the combined effects of turbulence-induced amplitude fluctuations and phase aberrations, it has been shown in [14] that the probability density function of fading amplitude $a_{k, i}=\left|\alpha_{k, i}\right|$ follows Rician distribution, i.e.,

$$
\begin{aligned}
f_{a_{k, i}}\left(a_{k, i}\right)= & \frac{2 a_{k, i}\left(1+K_{k, i}\right)}{\overline{a_{k, i}^{2}}} e^{-K_{k, i}-\frac{\left(1+K_{k, i}\right) a_{k, i}^{2}}{a_{k, i}^{2}}} \\
& \times I_{0}\left(2 a_{k, i} \sqrt{\frac{K_{k, i}\left(1+K_{k, i}\right)}{\overline{a_{k, i}^{2}}}}\right),
\end{aligned}
$$

where $I_{n}(\cdot)$ is the $n^{\text {th }}$-order modified Bessel function of the first kind [18] and $K_{k, i}$ is the ratio of the strength of the coherent component to the noncoherent one of the detected field. It can be noted from [14] that both $\overline{a_{k, i}^{2}}$ and $K_{k, i}$ are functions of log-amplitude variance, the residual phase variance (i.e., after phase compensation) [19], and the number of statistically independent coherent cells in the receiveaperture plane. We assume that the channel coefficients within each hop are independent and identically distributed (i.i.d), i.e., $K_{k, i}=K_{k}$ and $\overline{a_{k, i}^{2}}=\overline{a_{k}^{2}}$ for $1 \leq i \leq m_{k}$. Sub-channels from one hop to another hop are assumed to be mutually independent, but not necessarily identical.

Besides the effect of small-scale fading, we also consider the effect of path loss. The path loss in the $k^{\text {th }}$ hop is given by $l\left(Z_{k}\right)=\left(S_{t} S_{r} e^{-\sigma Z_{k}}\right) /\left(\lambda Z_{k}\right)^{2}$ where $S_{t}$ is the transmitter's aperture area, $\lambda$ is the optical wavelength, and $\sigma$ is the atmospheric attenuation coefficient [1].

\section{Derivation of Outage Probability}

Atmospheric turbulence results in a very slowly varying fading channel. For such quasi-static channels, the outage probability is an appropriate metric to evaluate the system performance. The outage probability at a given transmission rate $R$ is defined as [20] $P_{\text {out }}(R)=\operatorname{Pr}\left\{\gamma<\gamma_{R}\right\}$ where $\gamma$ is the instantaneous SNR of the underlying system and $\gamma_{R}=2^{R}-1$ denotes the threshold SNR that is required to support the rate $R$. In the multi-hop transmission scheme with decode-and-forward relaying under consideration, the outage of any intermediate sub-channels causes a failure in the source-destination communication. Hence, the outage probability for the end-to-end scheme, denoted by $P_{\text {out }}^{S D}$, is given by

$$
\begin{aligned}
P_{\text {out }}^{S D} & =\operatorname{Pr}\left\{\bigcup_{k=1}^{M+1}\left(\gamma_{k}<\gamma_{R}\right)\right\} \\
& =1-\operatorname{Pr}\left\{\bigcap_{k=1}^{M+1}\left(\gamma_{k} \geq \gamma_{R}\right)\right\}=1-\prod_{k=1}^{M+1}\left(1-P_{\text {out }}^{k}\right),
\end{aligned}
$$

where $P_{o u t}^{k}$ is the outage probability of the $k^{\text {th }}$ intermediate single input-multiple output (SIMO) link with $m_{k}$ receive apertures, and $\gamma_{k}=P_{X_{k}} / P_{N_{k}}$ is the output SNR at the $k^{\text {th }}$ node. $P_{X_{k}}$ and $P_{N_{k}}$ are, respectively, the average received signal power and the noise power at the $k^{\text {th }}$ node. Using (1), $P_{X_{k}}$ is calculated as

$$
P_{X_{k}}=\left(2 \Re^{2} A_{L}^{2} \frac{S_{r}}{m_{k}}\right) \frac{P_{r_{k}}^{2}}{m_{k}} \sum_{i=1}^{m_{k}} a_{k, i}^{2},
$$

where $P_{r_{k}}=\overline{\left|u_{k}(t)\right|^{2}} S_{r}$ is the average received optical power in the absence of the fading. $P_{r_{k}}$ is related to the average transmit optical power per transmit aperture $\left(P_{s}\right)$ by $P_{r_{k}}=L_{k} P_{s}$ where $L_{k}$ denotes the path loss of the $k^{\text {th }}$ hop normalized with respect to the end-to-end link distance, i.e., $L_{k}=l\left(Z_{k}\right) / l\left(Z_{S D}\right)$. On the other hand, the noise power within the signal bandwidth of $B_{s}$ is given by [16]

$$
P_{N_{k}}=2 B_{s} e \Re A_{L}^{2} S_{r} / m_{k},
$$

where $e$ is the electron's charge. Based on (4) and (5), we have

$$
\gamma_{k}=\frac{L_{k} \rho}{m_{k}(M+1)} \sum_{i=1}^{m_{k}} a_{k, i}^{2}
$$

where $\rho=\Re P_{t} / e B_{s}$ is the total turbulence-free and path loss-free SNR of the system. Noting that $a_{k, i}$ is i.i.d. with parameters $K_{k}$ and $\overline{a_{k}^{2}}$, we can obtain $P_{o u t}^{k}$ and therefore have the outage probability for the end-to-end scheme from (3) as

$$
P_{\text {out }}^{S D}=1-\prod_{k=1}^{M+1} Q_{m_{k}}\left(\sqrt{2 m_{k} K_{k}}, \sqrt{\frac{2 m_{k}\left(1+K_{k}\right)(M+1) \gamma_{R}}{L_{k} \rho \overline{a_{k}^{2}}}}\right),
$$

where $Q_{m_{k}}(\cdot, \cdot)$ is the generalized Marcum $Q$-function of order $m_{k}$ [21].

\section{Derivation of Diversity-Multiplexing TRADEOFF AND DIVERSITY GAIN}

In this section, we derive the diversity-multiplexing tradeoff and diversity gain for the FSO relaying system under consideration. 


\section{A. Diversity-Multiplexing Tradeoff (DMT)}

DMT is an information-theoretic performance tool to bridge diversity and multiplexing gains which, respectively, quantify the link reliability and the spectral efficiency. Conventional definitions of multiplexing and diversity gains apply to the asymptotically high SNRs. They are, respectively, given by [15] $r_{\text {asym }}=\lim _{\rho \rightarrow \infty} R(\rho) / \log \rho$ and $d_{\text {asym }}=$ - $\lim _{\rho \rightarrow \infty} \log P_{\text {out }}(R, \rho) / \log \rho$ where $R(\rho)$ is the target data rate at a given $\operatorname{SNR}(\rho)$ and $P_{\text {out }}(R, \rho)$ is the outage probability. These definitions indicate the tradeoff between the data rate (quantified by the multiplexing gain) and the reliability (quantified by the diversity gain) of the system in high-SNR regime.

Definitions for multiplexing and diversity gains for finiteSNR regimes have been further introduced which are particularly useful in evaluating these gains at practical SNR values [22]. The multiplexing and diversity gains in finiteSNR regime are, respectively, defined as

$$
\begin{gathered}
r=\frac{R(\rho)}{\log (1+\rho)}, \\
d(r, \rho)=-\frac{\partial \log P_{\text {out }}(r, \rho)}{\partial \log \rho} .
\end{gathered}
$$

It can be readily checked that the definitions of finite SNR diversity and multiplexing gains are consistent with the asymptotical ones; that is, $\lim _{\rho \rightarrow \infty} d(R, \rho)=d_{\text {asym }}$ and $\lim _{\rho \rightarrow \infty} r=$ $r_{\text {asym. }}$.

When the transmission rate $R$ is a function of the SNR as $R(\rho)=r \log (1+\rho)$, the threshold SNR $\gamma_{R}$ depends on $r$ and $\rho$ as $\gamma_{R}=2^{R}-1=(1+\rho)^{r}-1$. Replacing this in (7) yields

$$
P_{\text {out }}^{S D}(r, \rho)=1-\prod_{k=1}^{M+1} Q_{m_{k}}\left(\zeta_{k}, \beta_{k}\right)
$$

where $\beta_{k}=\sqrt{2 m_{k}\left(1+K_{k}\right)(M+1)\left((1+\rho)^{r}-1\right) /\left(L_{k} \rho \overline{a_{k}^{2}}\right)}$ and $\zeta_{k}=\sqrt{2 m_{k} K_{k}}$. From (9) and (10), the finite-SNR DMT of the end-to-end scheme is given by

$$
\begin{aligned}
d^{S D}(r, \rho) & =-\frac{\rho}{P_{\text {out }}^{S D}(r, \rho)} \frac{\partial P_{\text {out }}^{S D}(r, \rho)}{\partial \rho} \\
& =\frac{\rho}{1-\prod_{k=1}^{M+1} Q_{m_{k}}\left(\zeta_{k}, \beta_{k}\right)} \\
& \times \sum_{k=1}^{M+1}\left(\frac{\partial Q_{m_{k}}\left(\zeta_{k}, \beta_{k}\right)}{\partial \beta_{k}} \frac{\partial \beta_{k}}{\partial \rho} \prod_{\substack{j=1 \\
j \neq k}}^{M+1} Q_{m_{j}}\left(\zeta_{j}, \beta_{j}\right)\right) .
\end{aligned}
$$

After some mathematical manipulations, we obtain $d^{S D}(r, \rho)$ as

$$
\begin{aligned}
& d^{S D}(r, \rho)=\left(1-\frac{r \rho(1+\rho)^{r-1}}{(1+\rho)^{r}-1}\right) \\
& \times \frac{\sum_{k=1}^{M+1}\left(\frac{\beta_{k}^{m_{k}+1}}{\zeta_{k}^{m_{k}-1}} e^{-\frac{\left(\beta_{k}^{2}+\zeta_{k}^{2}\right)}{2}} I_{m_{k}-1}\left(\zeta_{k} \beta_{k}\right) \prod_{\substack{j=1 \\
j \neq k}}^{M+1} Q_{m_{j}}\left(\zeta_{j}, \beta_{j}\right)\right]}{2\left[1-\prod_{k=1}^{M+1} Q_{m_{k}}\left(\zeta_{k}, \beta_{k}\right)\right]} .
\end{aligned}
$$

It is also possible to show that $d^{S D}(r, \rho)$ can be written in terms of the finite-SNR DMT of each SIMO sub-channel as

$$
d^{S D}(r, \rho)=\frac{1-P_{\text {out }}^{S D}}{P_{\text {out }}^{S D}} \sum_{k=1}^{M+1} d^{k}(r, \rho) \frac{P_{\text {out }}^{k}}{1-P_{\text {out }}^{k}},
$$

where the finite-SNR DMT of the $k^{\text {th }}$ SIMO sub-channel is given by [16]

$$
\begin{aligned}
d^{k}(r, \rho) & =\left(1-\frac{r \rho(1+\rho)^{r-1}}{(1+\rho)^{r}-1}\right) \\
& \times \frac{\beta_{k}^{m_{k}+1}}{\zeta_{k}^{m_{k}-1}} \frac{e^{-\frac{\left(\beta_{k}^{2}+\zeta_{k}^{2}\right)}{2}} I_{m_{k}-1}\left(\zeta_{k} \beta_{k}\right)}{2\left[1-Q_{m_{k}}\left(\zeta_{k}, \beta_{k}\right)\right]} .
\end{aligned}
$$

For a sanity check, the asymptotic behavior of the derived DMT at high SNR is investigated in the Appendix. It is shown that

$$
\lim _{\rho \rightarrow \infty} d^{S D}(r, \rho)=\hat{m}(1-r), \quad 0 \leq r \leq 1
$$

where $\hat{m}=\min _{k}\left\{m_{k}\right\}$. This result agrees with the asymptotic DMT of the clustered multi-hop relay channels analyzed in the context of radio-frequency (RF) communication [23]. It demonstrates that at high-SNR regime, the DMT of the end-to-end scheme is determined by the minimum of the underlying sub-channels' DMT values throughout the range of the multiplexing gain.

\section{B. Finite SNR Diversity Gain for a Fixed Transmission Rate}

In this section, we derive the finite-SNR diversity gain when the transmission rate $R$ is fixed, i.e., it does not vary with SNR. Note that under this assumption, $\gamma_{R}$ is a constant and independent of $\rho$. Therefore, the finite-SNR diversity gain of the end-to-end scheme for a fixed $R$ is given by

$$
d^{S D}(R, \rho)=-\frac{\rho}{P_{\text {out }}^{S D}(R, \rho)} \frac{\partial P_{\text {out }}^{S D}(R, \rho)}{\partial \rho} .
$$

Note that (17) is similar to (11) except the fact that the data rate $R$ is now a constant. Therefore, solving (17) yields an expression of the same form as (12) in which $\beta_{k}$ is now given by $\beta_{k}=\sqrt{2 m_{k}\left(1+K_{k}\right)(M+1) \gamma_{R} /\left(L_{k} \rho \overline{a_{k}^{2}}\right)}$. Consequently, we obtain

$$
\begin{aligned}
& d^{S D}(R, \rho)= \\
& \frac{\sum_{k=1}^{M+1}\left(\frac{\beta_{k}^{m_{k}+1}}{\zeta_{k}^{m_{k}-1}} e^{-\frac{\left(\beta_{k}^{2}+\zeta_{k}^{2}\right)}{2}} I_{m_{k}-1}\left(\zeta_{k} \beta_{k}\right) \prod_{\substack{j=1, j \neq k}}^{M+1} Q_{m_{j}}\left(\zeta_{j}, \beta_{j}\right)\right)}{2\left[1-\prod_{k=1}^{M+1} Q_{m_{k}}\left(\zeta_{k}, \beta_{k}\right)\right]}
\end{aligned}
$$

From (13) and (18), it can be readily checked that

$$
d^{S D}(r, \rho)=\left.d^{S D}(R, \rho)\right|_{R=r \log (1+\rho)} \times\left(1-\frac{r \rho(1+\rho)^{r-1}}{(1+\rho)^{r}-1}\right) \text {. }
$$

Although the derivations of the finite-SNR DMT and diversity gain at a fixed rate are very similar, they represent two individual performance metrics at practical SNR values and they are not equivalent for any specific value of multiplexing 


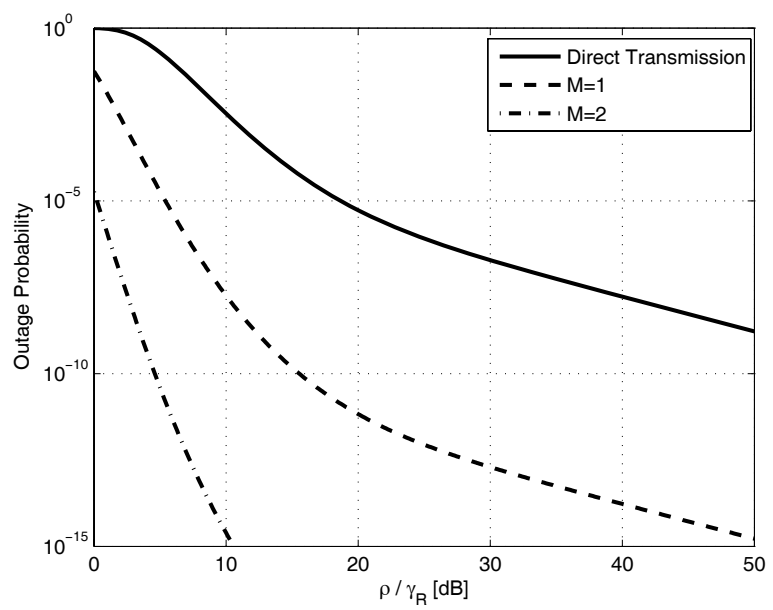

Fig. 1. Outage probability for coherent FSO systems with $M=1,2$, and $m=1$.

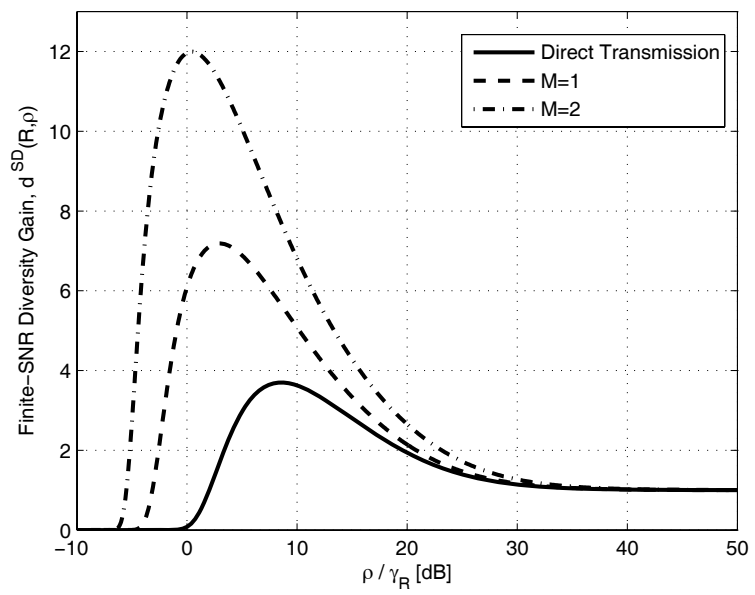

Fig. 2. Finite-SNR diversity gain at a fixed transmission rate $R$ for $M=1,2$, and $m=1$.

gain $r$. However, in high-SNR regime, it is expected that the diversity gain at a fixed rate $R$ will be equal to the DMT at $r=0$ [15]. It can be readily checked from (16) that the asymptotic DMT at $r=0$ is given by $\hat{m}$ while from (18) we have $\lim _{\rho \rightarrow \infty} d^{S D}(R, \rho)=\hat{m}$ as expected. This result demonstrates that, in high-SNR regime, the diversity order of the end-to-end transmission is restricted by the minimum number of receive apertures of each hop.

\section{Numerical Results AND Discussions}

In this section, we present numerical results for outage probability, DMT and diversity gain using the analytical expressions derived in the previous sections. We consider an FSO system with the wavelength $\lambda=1.55 \mu \mathrm{m}$ operating in turbulence conditions with refractive index structure constant $C_{n}^{2}=1 \times 10^{-14} \mathrm{~m}^{-2 / 3}$ and attenuation of $0.44 \mathrm{~dB} / \mathrm{km}$ (i.e., $\sigma \approx 0.1$ ). The link range is $Z_{S D}=5 \mathrm{~km}$ and the total receive aperture diameter at each node is $D=25 \mathrm{~cm}$. In the first three figures (Figs. 1-3), to demonstrate the spatial advantages extracted from multi-hop transmission in FSO systems, we

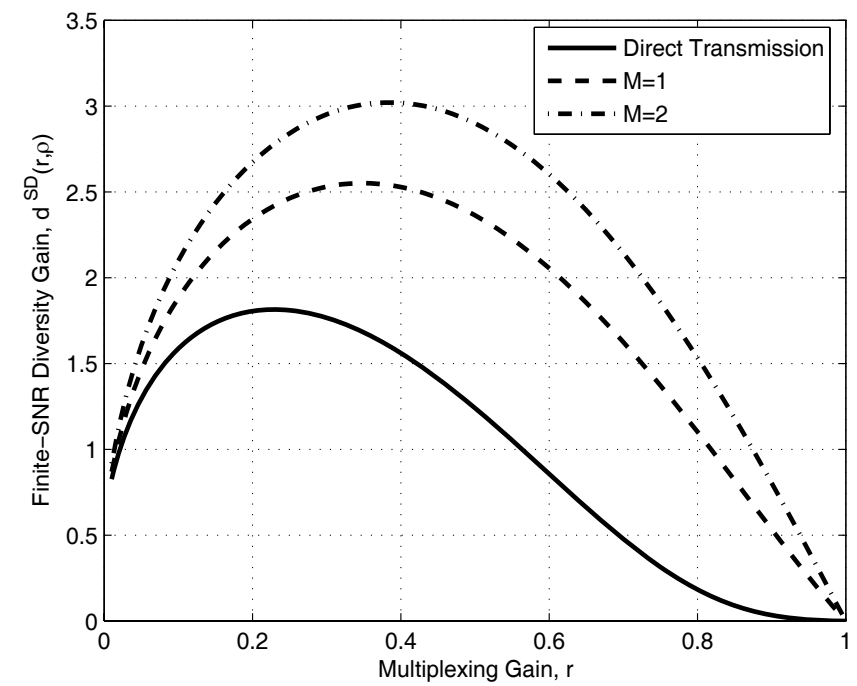

Fig. 3. Finite-SNR DMT for $M=1,2$ and $m=1$ at a fixed $\mathrm{SNR}=10 \mathrm{~dB}$.

assume each node is equipped with single receive aperture i.e., $m_{k}=1,1 \leq k \leq M+1$, and therefore, no receive diversity is available. At each receive-aperture, $J=6$ Zernike modes [19] are compensated. We assume a symmetric scenario for these figures, i.e., $Z_{k}=Z_{S D} /(M+1), K_{k}=K$ and $\overline{a_{k}^{2}}=\overline{a^{2}} \forall k$. Fig. 1 illustrates the outage probability given by (7) versus normalized SNR $\left(\rho / \gamma_{R}\right)$ for $M=1,2$. For comparison purposes, the outage probability of the direct transmission is also presented in this figure. It is observed that multi-hop transmission exploits the distance-dependent characteristic of fading variance and significantly improves the system performance. Specifically, at a target outage probability of $10^{-8}$, impressive performance improvements of $31.5 \mathrm{~dB}$ and $39.2 \mathrm{~dB}$ are, respectively, observed for one and two relays in comparison to the benchmark direct transmission system.

Fig. 2 depicts the finite-SNR diversity gain given by (18) for a fixed transmission rate $R$. This actually corresponds to the negative slope of the outage probability curves illustrated in Fig. 1. It is observed that the maximum diversity gain occurs at a finite value of SNR. This is mainly due to the presence of the coherent component in the received field, similar to the phenomenon which is observed in Rician RF channels [22], [24]. Indeed, at moderate SNRs, the presence of the coherent component causes a steep drop in the outage probability and results in a peak in the finite-SNR diversity gain. As the SNR increases, the noncoherent (random) component begins to dominate the system performance and decreases the diversity gain to its asymptotical value which is equal to one in our case.

Fig. 3 illustrates the finite-SNR DMT given by (13) for various number of relays $M=1,2$ assuming a fixed $\mathrm{SNR}=$ $10 \mathrm{~dB}$. It is observed that multi-hop transmission provides diversity advantages throughout the range of the multiplexing gain. Especially, at high values of multiplexing gain, the diversity gain of the direct transmission approaches zero which can be avoided by the proposed multi-hop transmission.

Fig. 4 presents a performance comparison between multi- 


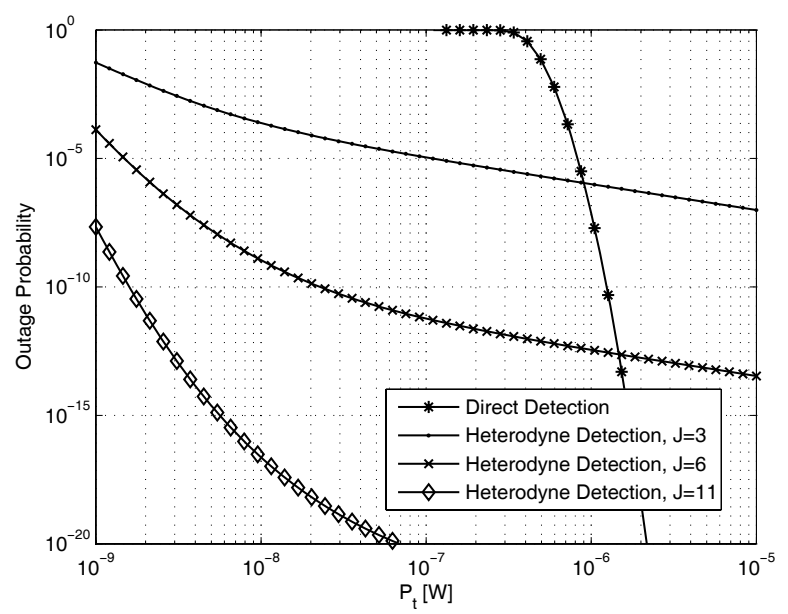

Fig. 4. Performance comparison of coherent and IM/DD multi-hop systems $(M=1$ and $m=1)$.

hop coherent and IM/DD systems assuming the deployment of single relay and single receive apertures. For IM/DD systems, it has been shown in [25] through experimental observations that the fading amplitude follows log-normal distribution even for a large receive-aperture. Under the assumption of background noise-limited receiver and following similar steps in [8], we can obtain the outage probability for the multi-hop IM/DD scheme as

$$
\begin{aligned}
& P_{\text {out }, \mathrm{IM} / \mathrm{DD}}^{S D}= \\
& 1-\prod_{k=1}^{M+1}\left[1-Q\left(\frac{\ln \left(\frac{\Re L_{k} P_{t}}{(M+1) \sqrt{2 e \Re B_{s} P_{b} \gamma_{R}}}\right)-2 \sigma_{\chi}^{2}(D)}{2 \sigma_{\chi}(D)}\right)\right],
\end{aligned}
$$

where $Q(\cdot)$ denotes the Gaussian $Q$-function [21], $P_{b}$ is the background noise power in each receiver's aperture, and $\sigma_{\chi}^{2}(D)$ is the log-amplitude variance of a receive-aperture with diameter $D$ [26]. It is assumed that $B_{s}=1 \mathrm{GHz}, R=2$ bits/symbol, and $P_{b}=1 \mathrm{~mW}$. Performance plots in Fig. 4 are provided under the assumption of same transmit power $P_{t}$ for both systems. We observe that, for small values of $P_{t}$, coherent detection brings better performance compared to the noncoherent one, even for low order of compensation $(J=3)$. Performance curves for coherent and noncoherent systems intersect for larger values of $P_{t}$ since the infinite diversity order for a Rician channel (in coherent system) is just one while for a lognormal channel (in IM/DD system) is infinity. However, if the degree of compensation increases at the cost of higher complexity, considerable power gains can be achieved by coherent detection for a desired value of outage probability.

In the following, we consider a coherent FSO system with multiple receive apertures to further exploit the receive diversity advantages. It is assumed that $J=3$ modes are compensated in each receive-aperture. The system is equipped with two relay nodes (i.e., $M=2$ ) distributed between the source and the destination with distances $Z_{1}=1 \mathrm{~km}$, $Z_{2}=2.5 \mathrm{~km}$, and $Z_{3}=1.5 \mathrm{~km}$. We consider three different systems based on the number of receive apertures at each node. In the first system (S1), we assume $m_{1}=m_{2}=m_{3}=2$.

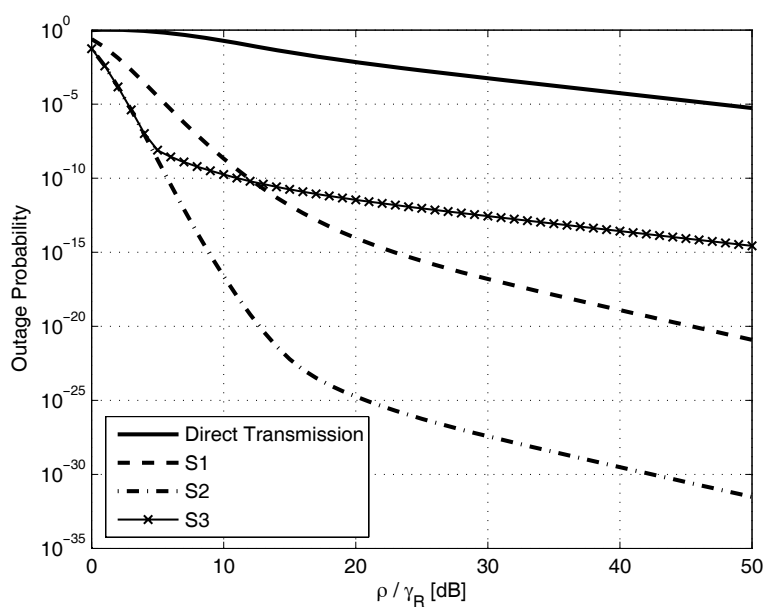

Fig. 5. Outage probability of asymmetrical coherent systems S1, S2 and S3.

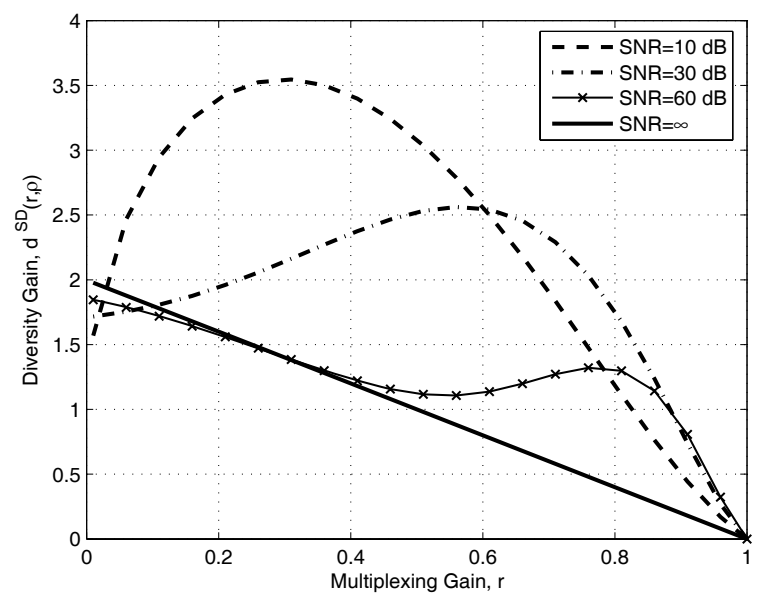

Fig. 6. DMT of system S2 for different values of SNR.

In the second system (S2), we have $m_{1}=2, m_{2}=3$, and $m_{3}=2$. Finally, in the third system (S3), we have $m_{1}=1$, $m_{2}=3$, and $m_{3}=2$.

Fig. 5 depicts the outage probability versus normalized SNR $\left(\rho / \gamma_{R}\right)$ for these three systems. A common observation for all systems is the impressive performance improvements through multi-hop transmission encoupled with receive diversity. For small values of SNR (SNR $<5 \mathrm{~dB}$ ), the outage performance of S2 and S3 is the same while both outperform S1. In this range, the second sub-channel (i.e., the longest hop which experiences the poorest fading conditions) has the worst outage performance and acts as a bottleneck in the performance of the end-to-end data transmission. Hence, increasing the number of receive-apertures at the second relay from two (in S1) to three (in S2 and S3) improves the performance. On the other hand, as SNR increases, performance curves of S2 and S3 deviate from each other because the noncoherent component of the Rician channel begins to dominate the system performance and the deployment of receive diversity at the first relay results in S2's additional performance improvement. In this regime, the performance of S3 is restricted by its first relay which has only one receive aperture. It can be readily checked from (16) 
that the asymptotic diversity gain of S1, S2 and $\mathrm{S} 3$ is given by two, two, and one, respectively.

Fig. 6 depicts the finite-SNR DMT of S2 for various values of SNR. It is observed that, for finite values of SNR, the maximum diversity gain does not occur at zero multiplexing gain. Similar to our observations in Fig. 2, this is due to the presence of coherent component. When SNR increases, the noncoherent component begins to dominate the system performance and the DMT curve gradually approaches its asymptotic value. Recall that the number of receive apertures in each hop is given by $m_{1}=2, m_{2}=3$, and $m_{3}=2$. It is observed that as SNR increases gradually, the DMT performance approaches $\min \{2,3,2\} \times(1-r)=2(1-r)$. Therefore, the asymptotic DMT of the end-to-end scheme is determined by the minimum of the DMT values of all subchannels throughout the range of the multiplexing gain.

\section{CONCLUSIONS}

In this paper, we have presented multi-hop relaying as a powerful fading mitigation tool for coherent FSO systems over atmospheric turbulence channels. We have derived the outage probability, finite-SNR DMT, and finite-SNR diversity gains taking into account the effects of both turbulenceinduced amplitude fluctuation and phase aberration. Our results quantify the performance gains in terms of diversity and multiplexing available through multi-hop relaying and yield impressive performance improvements over the conventional direct transmission.

\section{APPENDIX}

In this appendix, we derive the asymptotic value of DMT given by (16) starting from the finite SNR DMT expression of (13). It can be readily checked that when $\rho \rightarrow \infty$ we have $\beta_{k} \rightarrow \sqrt{B_{k} \rho^{r-1}}$, where $B_{k}=2 m_{k}\left(1+K_{k}\right)(M+1) /\left(L_{k} \overline{a_{k}^{2}}\right)$. Since $r<1$ [15], we observe $\lim _{\rho \rightarrow \infty} \beta_{k}=0, \forall k$. Consequently, we obtain

$$
\lim _{\rho \rightarrow \infty} d^{S D}(r, \rho)=\lim _{\beta_{k} \rightarrow 0} \frac{g_{1}}{2 g_{2}} \times \lim _{\rho \rightarrow \infty}\left(1-\frac{r \rho(1+\rho)^{r-1}}{(1+\rho)^{r}-1}\right),
$$

where we define $g_{1}$ and $g_{2}$ as

$$
g_{1}=\sum_{k=1}^{M+1}\left(\frac{\beta_{k}^{m_{k}+1}}{\zeta_{k}^{m_{k}-1}} e^{-\frac{\beta_{k}^{2}+\zeta_{k}^{2}}{2}} I_{m_{k}-1}\left(\zeta_{k} \beta_{k}\right) \prod_{\substack{j=1, j \neq k}}^{M+1} Q_{m_{j}}\left(\zeta_{j}, \beta_{j}\right)\right),
$$

$$
g_{2}=1-\prod_{k=1}^{M+1} Q_{m_{k}}\left(\zeta_{k}, \beta_{k}\right) .
$$

It is easy to see that the second limit term in (21) yields $1-r$. For the solution of the first limit expression, we first work with $g_{2}$. Replacing the Marcum $Q$-function by its series form and using the small argument form of the modified Bessel function of the first kind [21], we have

$$
\lim _{\beta_{k} \rightarrow 0} g_{2}=\lim _{\beta_{\hat{k}} \rightarrow 0} \frac{\left(\beta_{\hat{k}}^{2} / 2\right)^{\hat{m}}}{\hat{m} !} e^{-\frac{\zeta_{\hat{k}}^{2}+\beta_{\hat{k}}^{2}}{2}}
$$

where $\hat{m}=\min _{k}\left\{m_{k}\right\}$ and $\hat{k}=\arg \left(\min _{k}\left\{m_{k}\right\}\right)$. For $g_{1}$ in (22), first note that $\lim _{\beta_{j} \rightarrow 0} Q_{m_{j}}\left(\zeta_{j}, \beta_{j}\right)=1$ [21]. Therefore, we have

$$
\lim _{\beta_{k} \rightarrow 0} g_{1}=\lim _{\beta_{\hat{k}} \rightarrow 0} 2 \frac{\left(\beta_{\hat{k}}^{2} / 2\right)^{\hat{m}}}{(\hat{m}-1) !} e^{-\frac{\left(\zeta_{\hat{k}}^{2}+\beta_{\hat{k}}^{2}\right)}{2}} .
$$

Finally, replacing (24) and (25) in (21), we obtain the asymptotical DMT expression as given by (16).

\section{REFERENCES}

[1] R. M. Gagliardi and S. Karp, Optical Communications, 2nd edition. John Wiley \& Sons, 1995.

[2] D. Kedar and S. Arnon, "Urban optical wireless communication networks: the main challenges and possible solutions," IEEE Commun. Mag., vol. 42, no. 5, pp. S2-S7, May 2004.

[3] V. W. S. Chan, "Free-space optical communication," IEEE J. Lightwave Technol., vol. 24, no. 12, pp. 4750-4762, Dec. 2006.

[4] A. S. Acampora and S. V. Krishnamurthy, "A broadband wireless access network based on mesh-connected free-space optical links," IEEE Personal Commun., vol. 6, pp. 62-65, Oct. 1999.

[5] J. Akella, M. Yuksel, and S. Kalyanaraman, "Error analysis of multi-hop free-space optical communication," in Proc. IEEE International Conf. Commun., May 2005.

[6] T. A. Tsiftsis, H. G. Sandalidis, G. K. Karagiannidis, and N. C. Sagias, "Multihop free-space optical communications over strong turbulence channels," in Proc. IEEE International Conf. Commun., June 2006.

[7] G. K. Karagiannidis, T. A. Tsiftsis, and H. G. Sandalidis, "Outage probability of relayed free space optical communication systems," Electron. Lett., vol. 42, pp. 994-995, Aug. 2006.

[8] M. Safari and M. Uysal, "Relay-assisted free-space optical communication," IEEE Trans. Wireless Commun., vol. 7, no. 12, pp. 5441-5449, Dec. 2008.

[9] M. Safari and M. Uysal, "Diversity gain analysis of free-space optical communication systems," Canadian Conf. Electrical Comput. Eng., May 2008.

[10] E. Lee and V. Chan, "Diversity coherent and incoherent receivers for free-space optical communication in the presence and absence of interference," IEEE/OSA J. Opt. Commun. Netw. vol. 1, no. 5, pp. 463483, Oct. 2009.

[11] H. G. Sandalidis, T. A. Tsiftsis, and G. K. Karagiannidis, "Optical wireless communications with heterodyne detection over turbulence channels," IEEE J. Lightwave Technol., vol. 27, no. 20, pp. 4440-4445, Oct. 2009.

[12] N. Cvijetic, D. Qian, J. Yu, Y. K. Huang, and T. Wang, "Polarizationmultiplexed optical wireless transmission with coherent detection," IEEE J. Lightwave Technol., vol. 28, no. 8, pp. 1218-1227, Apr. 2010.

[13] Robert K. Tyson, Principles of Adaptive Optics. Academic Press, 1991.

[14] A. Belmonte and J. M. Kahn, "Performance of synchronous optical receivers using atmospheric compensation techniques," Opt. Express, vol. 16, no. 18, pp. 14151-14162, Sep. 2008.

[15] L. Zheng and D. N. C. Tse, "Diversity and multiplexing: a fundamental tradeoff in multiple antenna channels," IEEE Trans. Inf. Theory, vol. 49, no. 5, pp. 1073-1096, May 2003.

[16] S. M. Aghajanzadeh and M. Uysal, "Diversity-multiplexing tradeoff in multiple receiver coherent free-space optical systems," IEEE/OSA $J$. Opt. Commun. Netw., vol. 2, no. 12, pp. 1087-1093, Dec. 2010.

[17] G. R. Osche, Optical Detection Theory for Laser Applications. John Wiley \& Sons, 2002.

[18] F. Bowman, Introduction to Bessel Functions. Dover, 1958.

[19] R. J. Noll, "Zernike polynomials and atmospheric turbulence," J. Opt. Soc. Am., vol. 66, no. 3, pp. 207-211, 1976.

[20] E. Biglieri, J. Proakis, and S. Shamai, "Fading channels: information theoretic and communications aspects," IEEE Trans. Inf. Theory, vol. 44, no. 6, pp. 2619-2692, Oct. 1998.

[21] M. K. Simon and M.-S. Alouini, Digital Communication over Fading Channels, 2nd edition. John Wiley \& Sons, 2005.

[22] R. Narasimhan, "Finite-SNR diversity-multiplexing tradeoff for correlated Rayleigh and Ricean MIMO channels," IEEE Trans. Inf. Theory, vol. 52, no. 9, pp. 3965-3979, Sep. 2006.

[23] S. Yang and J. C. Belfiore, "Diversity of MIMO multi-hop relay channels," submitted to IEEE Trans. Inf. Theory, Aug. 2007 [arXiv:0708.0386v1]. 
[24] W. Y. Shin, S. Y. Chung, and Y. H. Lee, "Diversity-multiplexing tradeoff and outage performance for Ricean MIMO channels," IEEE Trans. Inf. Theory, vol. 54, no. 3, pp. 1186-1196, Mar. 2008.

[25] F. S. Vetelino, C. Young, L. C. Andrews, and J. Recolons, "Aperture averaging effects on the probability density of irradiance fluctuations in moderate-to-strong turbulence," Appl. Opt., vol. 46, no. 11, pp. 20992108, Apr. 2007.

[26] D. L. Fried, "Aperture averaging of scintillation," J. Opt. Soc. Am. vol. 57, no. 2, pp. 169-175, Feb. 1967.

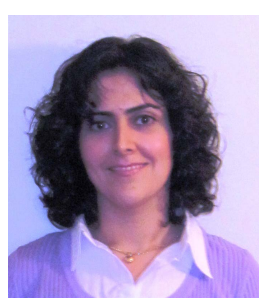

Sahar Molla Aghajanzadeh received the B.Sc. and the M.Sc. degree in electrical engineering from Sharif University of Technology, Tehran, Iran, in 2003 and 2006, respectively. She is currently a Ph.D. student at the department of electrical and computer engineering of the University of Waterloo, Waterloo, Canada. Her research interests include information theory and coding in free-space optical communication, cooperative communication, and fading mitigation techniques for wireless optical channels.

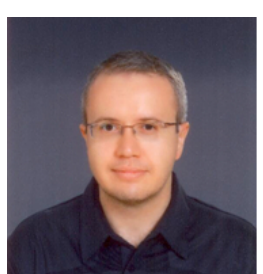

Murat Uysal was born in Istanbul, Turkey in 1973. He received the B.Sc. and the M.Sc. degree in electronics and communication engineering from Istanbul Technical University, Istanbul, Turkey, in 1995 and 1998, respectively, and the Ph.D. degree in electrical engineering from Texas A\&M University, College Station, Texas, in 2001. Since 2002, he has been with the Department of Electrical and Computer Engineering, University of Waterloo, Canada, where he is now an Associate Professor. He is currently on leave at Özyeğin University, Istanbul, Turkey. His general research interests lie in communications theory and signal processing for communications with particular emphasis on wireless applications. Specific research areas include MIMO communication techniques, spacetime coding, diversity techniques and coding for fading channels, cooperative communication, and free-space (wireless) optical communication. Dr. Uysal is an Associate Editor for IEEE TRANSACTIONS ON WIRELESS COMMUNICATIONS, IEEE COMMUNICATIONS LETTERS, and Wireless Communications and Mobile Computing (WCMC) Journal. In the past he served as a Guest Co-Editor for WCMC Special Issue on "MIMO Communications" (October 2004) and IEEE Journal on SELECTED AREAS IN COMMUNiCATIONS Special Issue on "Optical Wireless Communications" (December 2009). Over the years, he has served on the technical program committee of more than 70 international conferences and workshops in the communications area. $\mathrm{He}$ recently co-chaired IEEE ICC'07 Communication Theory Symposium and CCECE'08 Communications and Networking Symposium. He is currently serving as the Tutorial Chair of IEEE WCNC'11. Dr. Uysal is a Senior IEEE member. 